\title{
THE COMPUTATION OF CYCLIC PEPTIDE WITH PROLIN-PROLIN BOND AS FUSION INHIBITOR OF DENV ENVELOPE PROTEIN THROUGH MOLECULAR DOCKING AND MOLECULAR DYNAMICS SIMULATION
}

\author{
Arli Aditya Parikesit, Hilyatuzzahroh, Andreas S Nugroho, Amalia Hapsari, \\ Usman Sumo Friend Tambunan* \\ Department of Chemistry, Faculty of Mathematics and Science, University of Indonesia \\ * Corresponding author: usman@ui.ac.id
}

\begin{abstract}
A disease that caused by dengue virus (DENV) has become the major health problem of the world. Nowadays, no effective treatment is available to overcome the disease due to the level of dengue virus pathogeneses. A novel treatment method such as antiviral drug is highly necessary for coping with the dengue disease. Envelope protein is one of the non-structural proteins of DENV, which engaged in the viral fusion process. It penetrates into the host cell to transfer its genetic material into the targeted cell followed by replication and establishment of new virus. Thus, the envelope protein can be utilized as the antiviral inhibitor target. The fusion process is mediated by the conformational change in the protein structure from dimer to trimer state. The previous research showed the existing cavity on the dimer structure of the envelope protein. The existing ligand could get into cavity of the envelope protein, stabilize the dimer structure or hamper the transition of dimer protein into trimer. In this fashion, the fusion process can be prevented. The aim of this research is designing the cyclic peptide with prolin-prolin bond as fusion inhibitor of DENV envelope protein through molecular docking and molecular dynamics simulation. The screening of 3,883 cyclic peptides, each of them connected by prolin-prolin bond, through molecular docking resulted in five best ligands. The result showed that PYRRP was the best ligand. PAWRP was also chosen as the best ligand because it showed good affinity with protein cavity. Stability of ligand-protein complex was analyzed by molecular dynamics simulation. The result showed that PYRRP ligand was able to support the stability of DENV envelope protein dimer structure at $310 \mathrm{~K}$ and $312 \mathrm{~K}$. While PAWRP ligand actively formed complex with the DENV envelope protein at $310 \mathrm{~K}$ compared to $312 \mathrm{~K}$. Thus the PYRRP ligand has a potential to be developed as DENV fusion inhibitor.
\end{abstract}

Keywords: dengue, envelope protein, fusion process, cavity, cyclic peptide, molecular docking, molecular dynamics

\section{INTRODUCTION}

Disease that caused by dengue virus infection has become a global health problem, especially in tropical and subtropical regions such as Asia, Africa, and America. This infection has become endemic in more than 100 countries, including Indonesia. The World Health Organization estimates that there have been 50-100 million cases of dengue virus infection each year and as many as 2.5 billion people or $40 \%$ of the world's population are at risk of suffering this virus infection (WHO, 2012).

DENV, which is a positive single-chain RNA virus, belongs to the genus flavivirus and family Flaviviridae. DENV has four serotypes, namely DENV-1, DENV-2, DENV-3 and DENV4. Those serotypes had the same morphology and genome but show different antigens, thus a person can be infected more than once as there is no complete antibodies cross protection (Lindenbach \& Rice, 2007). Dengue virus RNA genome is a single strand Open Reading Frame (ORF) that encodes a polyprotein consisting of 3,391 amino acid residues forming 
three structural proteins, namely: C (capsid), PRM (pre-membrane), and E (envelope), and seven non-structural (NS) protein NS1, NS2A, NS2B, NS3, NS4A, NS4B, and NS5 (Zuo et al., 2009).

The fusion process occurs at the end of the fusion peptide loop of domain II. It occurs due to the movement around the hinge region between domains I-II border. This exposure led to the entry of the fusion peptide into the host cell membrane. At this transition stage, $E$ protein of the virus was linked with the host cell membrane. Both membranes are close together while the fusion of $\mathrm{E}$ protein triggers the formation of trimer (Kampmann et al., 2009). Previous studies related to the inhibition of DENV E protein by Modis et al., (2003) found the existence of a binding region (binding pocket) on the structure of the DENV-2 E protein. This pocket is occupied by a detergent molecule known as n-octyl- $\beta$-D-glucoside $(\beta-O G)$. Hence this pocket is called as $\beta-O G$ pocket and located in the hinge region between the envelope protein domains I-II. Transition of trimer formation is an important step in the entry of the dengue virus genome into the host cell (Li et al., 2008; Wang et al., 2009; Poh et al., 2009). Other related studies have also been conducted. Yennamali et al. (2009), have found the cavity site between domains I-III on the DENV-2 E protein. The site is only found in the structure of dimeric $E$ protein. The presence of a ligand that occupies this cavity stabilizes the dimer structure or inhibits $E$ protein trimerization, so that the fusion process can be disturbed. This research focused to find a compound that can occupy this cavity through virtual screening methods.

In our previous researches, we have designed disulfide cyclic peptide to target NS2BNS3 protease, RNA-dependent RNA polymerase and methyltransferase of NS5 protein of DENV (Idrus et al., 2012; Tambunan \& Alamudi, 2010; Tambunan et al., 2011a; Tambunan et al., 2011b). In this research, we designed cyclic peptides containing proline - proline bond and tested their affinities to target cavity of E protein via the molecular docking and molecular dynamics simulations. The selection of proline residues to form cyclic peptide was based on the ability of the secondary amine group of proline in reducing the rotational energy of proline - proline mediated-cyclization (Pohl et al., 2001).

\section{MATERIALS AND METHODS}

\section{Preparation of the envelope protein (E) DENV}

E Protein with 1OAN_A code was downloaded from the PDB and opened in the Molecular Operating Environment (MOE) 2008.10. E protein structure was adjusted and optimized by protonate 3D option in MOE [10]. The addition of hydrogen atoms was done by selecting the partial charge option. Energy minimization process was conducted by using the AMBER99 force field, gas phase solvation. The value of RMS gradient was set to 0.05 $\mathrm{kcal} / \mathrm{mol} \AA$ (Tambunan et al., 2011b).

\section{Design and preparation of cyclic peptide ligands}

The determination of cyclic peptide ligands as inhibitors was performed by analyzing the amino acid residues of the E protein cavity. The cyclic pentapeptide was formed with proline residues at its terminal. The combination of 7 polar amino acids (glycine, serine, threonine, cysteine, tyrosine, asparagine, and glutamine), 8 non polar amino acids (alanine, 
valine, leucine, isoleucine, phenylalanine, tryptophan, methionine, and proline), and 5 charged polar amino acids (lysine, arginine, histidine, aspartic acid, and glutamic acid) generated 3,883 cyclic pentapeptide molecules. The process of designing 3D structure of the ligand was done by using ChemSketch ACDLabs. Ligand optimization and minimization was done with MOE 2008.10. Optimization process was utilized by selecting the wash option and the partial charge option for all designed ligands. Energy minimization process was applied by using MMFF94x force field, gas phase solvation, and the value of RMS gradient $0.001 \mathrm{kcal}$ / mol Å (Tambunan et al., 2011b).

\section{Molecular Docking}

The docking between cyclic peptides as the ligands and $E$ protein was done by selecting simulation_dock option in MOE. Triangle matcher was selected as placement method. The utilized scoring function is London dG. The next appearance of the 100 best pose was refined based on force field parameter. The displayed result of overall docking process is the best pose.

\section{Molecular Dynamics Simulation}

Optimization and energy minimization of protein-ligand complexes were required before performing molecular dynamics simulation. Optimization was done by selecting the partial charge option. Energy minimization was applied by using the AMBER99 force field and born solvation. The RMS gradient value was set to $0.05 \mathrm{kcal} / \mathrm{mol}$ Å. Molecular dynamics simulation was performed using MOE by selecting simulation_dynamic option. The utilized parameters were NVT ensemble and the NPA algorithm. The applied force field was AMBER99.

\section{RESULTS AND DISCUSSION \\ Cavity analysis of E DENV protein}

The cavity of $E$ DENV protein was composed of 25 amino acid residues: residues 1 , 143-149, 156, 158, 178 and 295 of domain I, and residues 324, 333, 355-357, 359-366 of domain III (Yennamali et al., 2009) (Figure 1). Based on the visualization cavity with MOE, polar; uncharged polar; and non-polar amino acid residues selected as constituents of cyclic peptides (Figure 2).

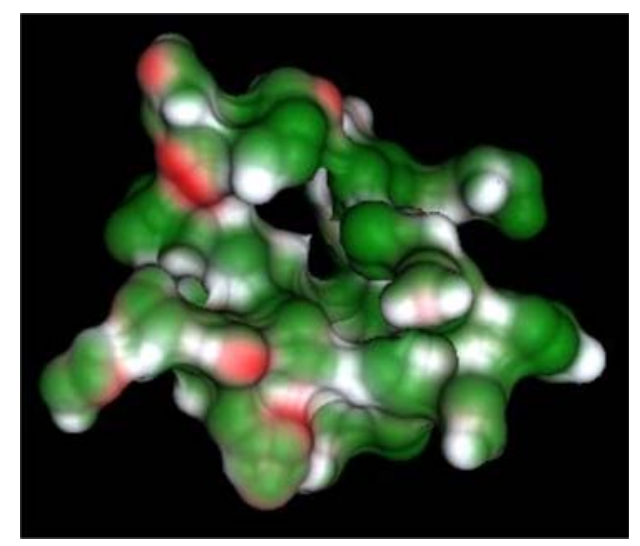

Figure 1. Cavity visualization of E protein DENV 


\section{Ligand screening throughmolecular docking process}

Table 1. Five best cyclic peptide ligandsaccording to molecular docking result

\begin{tabular}{lll}
\hline \multicolumn{1}{c}{ Ligand } & $\begin{array}{c}\Delta \mathrm{G}_{\text {binding }} \\
(\text { Kkal/mol })\end{array}$ & $\mathrm{pK} K_{\mathrm{i}}$ \\
\hline \hline PYRRP & -24.08 & 17.54 \\
\hline PAWRP & -20.29 & 14.78 \\
\hline PCWRP & -19.96 & 14.54 \\
\hline PFWRP & -18.94 & 13.79 \\
\hline PWPRP & -18.39 & 13.39 \\
\hline R1 Yennamalli* & -14.35 & 10.45 \\
\hline CLREC* & -12.36 & 9.00 \\
\hline A4 Kampmann* & -15.52 & 11.31 \\
\hline A5 Kampmann* & -14.69 & 10.69 \\
\hline C6 Wang* & -13.86 & 10.10 \\
\hline NITD448 Poh* & -11.567 & 8.423 \\
\hline *: Standard & &
\end{tabular}

Screening of 3,883 cyclic peptide ligands resulted in 5 best ligands based on the value of Gibbs free energy ( $\left.\ddot{A} G_{\text {binding }}\right)$ and the value of $p K_{i}($ Table1).

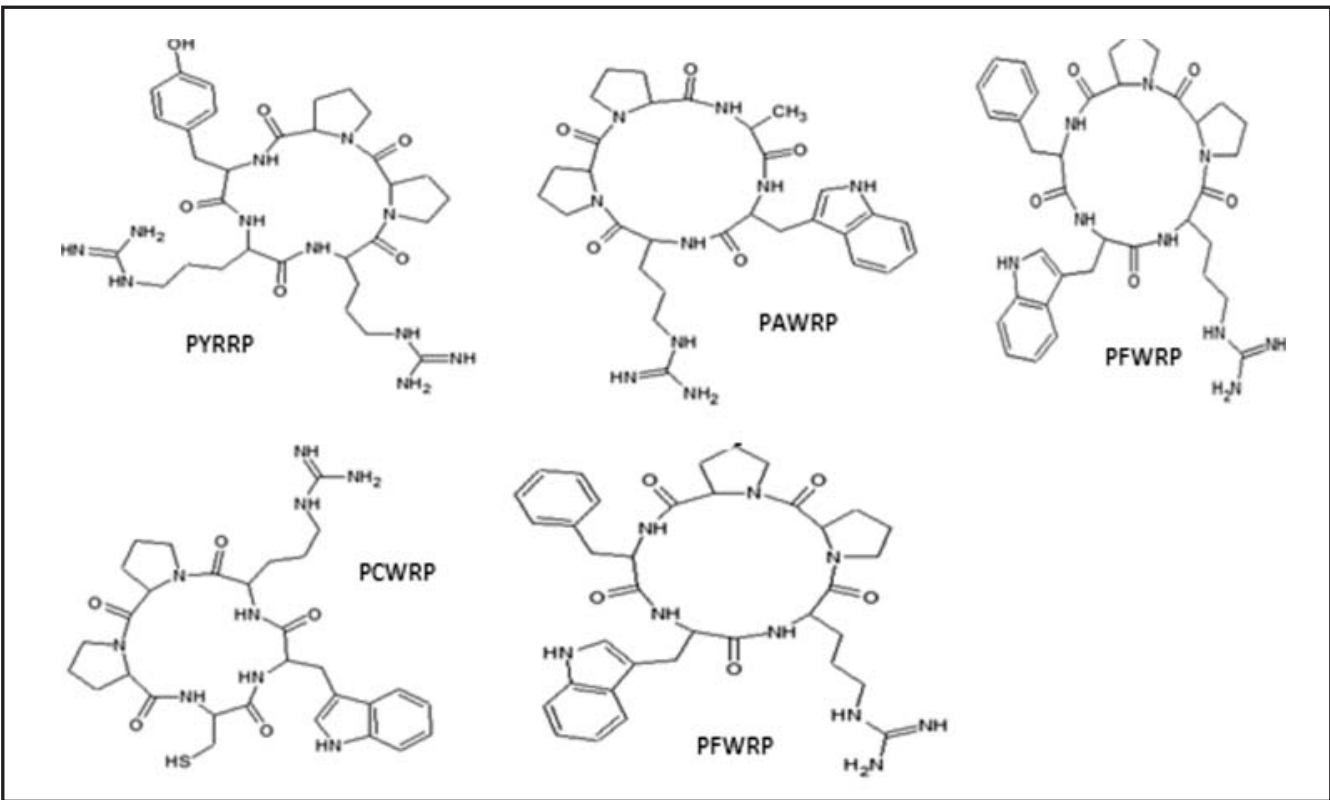

Figure 2. 2D images of the best cyclic peptide ligand screening results by molecular docking

According to the value of $\mathrm{AG}_{\text {binding }}$ and pKi, the PAWRP and PYRRP ligands showed better stability and good interaction with the protein than 6 standard ligands (Table 1). Stability and good interaction of the complex formation can be observed from the interaction of the hydrogen bonds.

\section{The results of molecular dynamics simulations}

Molecular dynamics simulation was used to analyze the conformational changes in the protein-ligand complex due to the influence of implicit solvent into the system. Therefore, the protein and ligand flexibility was set to born solvation (Alonso et al., 2006). There are three stages in the molecular dynamics simulations; the initialization, equilibration, and production stage. In the initialization phase, the initial state of the system was determined, including atomic coordinate, velocity and potential energy of the system. Initialization phase was done 
at a temperature of $300 \mathrm{~K}$ for $100 \mathrm{ps}$. The simulation was run until the system reached equilibration point that was indicated by the decline in the potential energy of system in order to achieve stability (Nurbaiti, 2009.). Production stage of the simulation produced trajectory. The obtained trajectory is a coordinate that was formed by taking the data changes in the state of each atom versus time due to the influence of temperature.

The second stage of the production of protein-ligand complexes (PYRRP and PAWRP) was performed at $310 \mathrm{~K}$ and $312 \mathrm{~K}$, which represent normal and fever body temperature, respectively. The production stage is divided into three stages, namely heating, the main simulation, and cooling. The heating and cooling stages wereconducted to find the lowest energy conformation of the complex. The result of the production phase at $310 \mathrm{~K}$ indicated that the two ligands, PYRRP and PAWRP, retained the hydrogenbond interaction with the envelope cavity until the end of the cooling stage. During the early stage of initialization until the end of 5000 ps simulation stage, the PYRRP and PAWRP ligands still interacted with the binding site residues of envelope protein cavity.

The result of the production phase at $312 \mathrm{~K}$ also showed that both ligands, PYRRP and PAWRP, also retained interaction with the cavity on the DENV envelope protein until the end of the cooling stage. During the initialization process until the end of $5000 \mathrm{ps}$ simulation stage, the PAWRP and PYRRP binding position were remain consistent with the binding site residues of envelope protein cavity. Hydrogen bonds between the PYRRP ligand and Glu A360, Asp A362, and Ser A363 residues of the envelope cavity remained stable until the end of the simulation. The magnitude of the protein-ligand complex conformational changes due to the influence of implicit solvent and temperature can be studied from the curve of its simulation time versus RMSD (Root Mean Square Deviation).

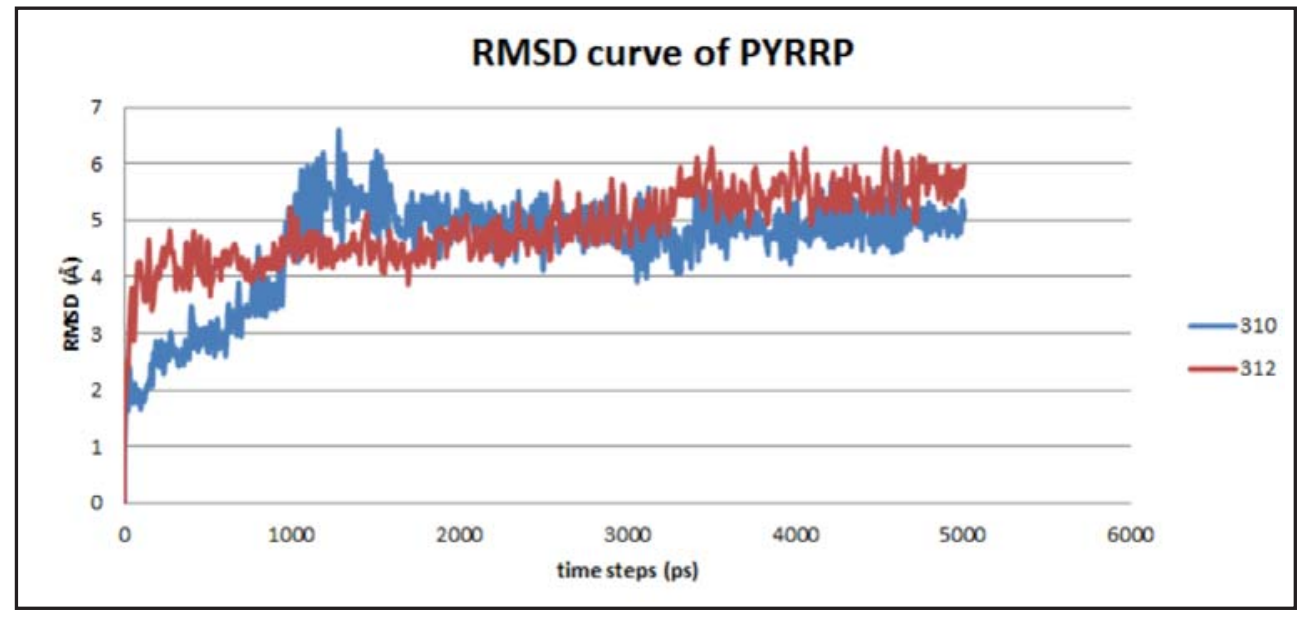

Figure 3. The relation between RMSD value of protein-ligand PYRRP complex and time steps during the 5000-ps-molecular-dynamics-simulation

The RMSD curve of protein-PYRRP complex showed that there were fluctuations during $1000-1500 \mathrm{ps}$ simulation at $310 \mathrm{~K}$. However, the complex maintained its stability at $310 \mathrm{~K}$ and $312 \mathrm{~K}$ in the interval of 2500-5000 ps simulation (Figure 3). Hence, the PYRRP ligand was able to maintain a stable interaction with the envelope cavity at both tested temperature.

RMSD curve of protein-PAWRP complex at $312 \mathrm{~K}$ has a smallerRMSDvalue than it has at $310 \mathrm{~K}$ (Figure 4). This suggests that there are more conformational changes of complex 
occur at $310 \mathrm{~K}$ rather than at $312 \mathrm{~K}$. Therefore, the complex between PAWRP and envelope protein is more stable at 310 than at $312 \mathrm{~K}$.

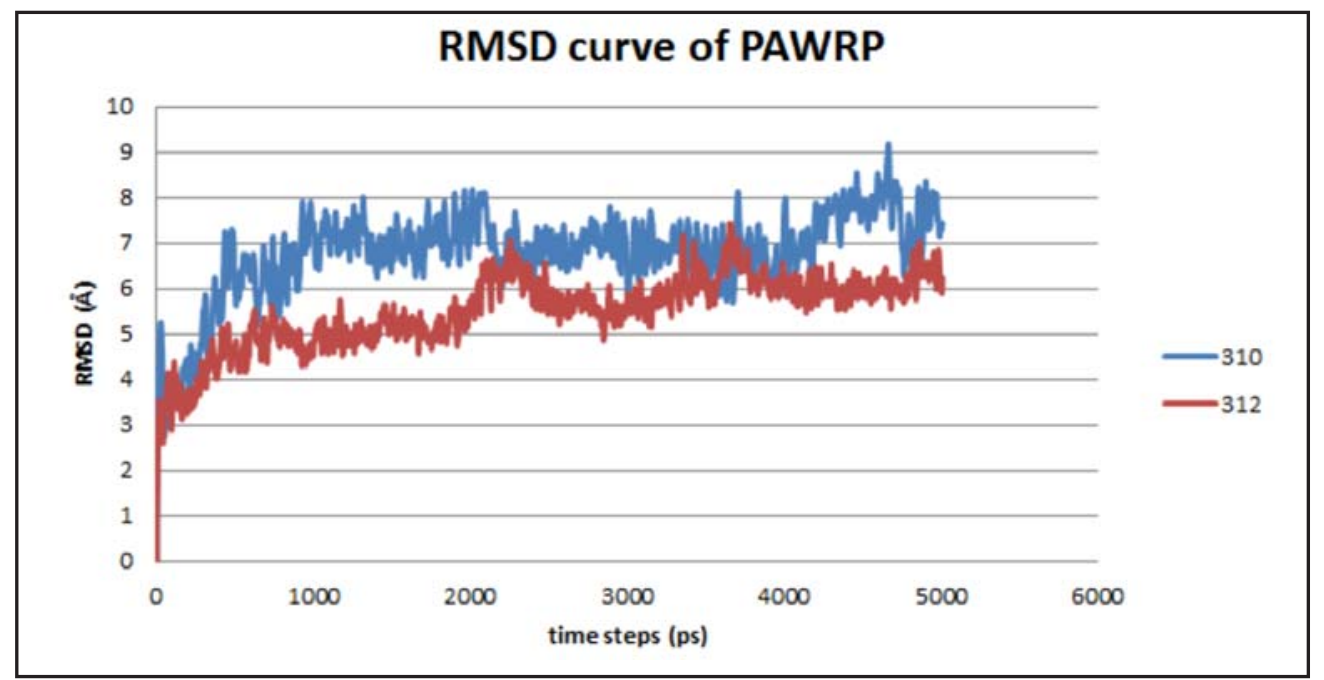

Figure 4. The relation between RMSD value of protein-ligand PAWRP complex and time steps during the 5000-ps-molecular-dynamics-simulation

\section{CONCLUSION}

The screening by means of molecular docking process generated five ligands (PYRRP, PAWRP, PCWRP, PFWRP, and PWPRP), which have better affinity (as indicated by their $\ddot{A}_{\text {binding }}$ and $\mathrm{pKi}$ ) with envelope cavity than the standards. PYRRP and PAWRP were the best top two ligandsamong all. During molecular dynamics simulation, both ligands were able to maintain interaction with DENV envelope protein cavity until the end of 5000-pssimulation, either at $310 \mathrm{~K}$ or at $312 \mathrm{~K}$. It was indicated from RMSD analysis that the PYRRP ligand was able to maintain the stability of protein-ligand complex at both tested temperature, while the PAWRP ligand was more reactive at $310 \mathrm{~K}$ than at $312 \mathrm{~K}$. Therefore, PYRRP ligand has potential to be developed furtheras a DENV fusion inhibitor.

\section{ACKNOWLEDGEMENT}

The authors would like to thank Hibah Riset Unggulan UI DRPM/RII/224/RU-UI/2013 for supporting this research. Usman Sumo Friend Tambunan supervised this research, Andreas S. Nugroho and Amalia Hapsari worked on the technical details; Arli Aditya Parikesit prepared the English manuscript and Hilyatuz Zahrah re-verified the data analysis, as well as proof reading. The authors would also thank to Dr.Syarifuddin Idrus for the technical assistance.

\section{REFERENCES}

WHO. 2012. Dengue and Dengue Haemorrhagic Fever. World Health Organization. Lindenbach, B.D., and C.M. Rice. 2007. Flaviridae: The Viruses and Their Replication.In Fundamental Virology. Knipe, D.M. \& P.M. Howley (eds). pp. 991"1041. Lippincott. Zuo, Z., et al. 2009. Mechanism of NS2B-Mediated Activation of NS3pro in Dengue Virus: Molecular Dynamics Simulation and Bioassays. Virology Journal,Vol.83(2):1060-1070. 
Kampmann, T., et al. 2009. In silico Screening of Small Molecule Libraries using The Dengue Virus E Protein has Identified Compounds with Antiviral Activity Againts Multiple Flaviviruses.Antiviral Research, Vol.84:234"241.

Modis, Y., et al. 2003. Ligand-binding Pocket in the Dengue Virus Envelope Glycoprotein. Proc Natl Acad Sci USA, Vol.100(12): 6986-91.

Li, Z., et al. 2008. Design, Synthesis, and Biological Evaluation of Antiviral Agents Targeting Flavivirus Envelope Protein. J MedChem, Vol.51(15):4660"4671.

Wang, Q.Y., et al. 2009. A Small-Molecule Dengue Virus Entry Inhibitor. Antimicrobial Agents and Chemotherapy, Vol.53(5):1823-1831.

Poh, M.K., et al. 2009. A Small Molecule Fusion Inhibitor of Dengue Virus. Antiviral Research, Vol.84:260"266.

Yennamali, R., et al. 2009. Identification of Novel Target Sites and an Inhibitor of The Dengue Virus E Protein. J. Computer Aided Mol,Vol.23:333-341.

Idrus, S., et al. 2012. Designing cyclopentapeptide inhibitor as potential antiviral drug for dengue virus NS5 methyltransferase. Bioinformation, Vol.8:348-352.

Tambunan, U.S.F. and Alamudi S. 2010. Designing cyclic peptide inhibitor of dengue virus NS3-NS2B protease by using molecular docking approach. Bioinformation,Vol.5(6): 250-254.

Tambunan, U.S.F., et al. 2011. Molecular Dynamics Simulation of DENV RNA-Dependent RNA-Polymerase with Potential Inhibitor of Disulfide Cyclic Peptide. Online Journal of Biological Sciences, Vol.11(2): 48-62.

Tambunan, U.S.F., et al. 2011. Computational Design of Disulfide Cyclic Peptide as Potential Inhibitor of Complex NS2B-NS3 Dengue Virus Protease. African Journal of Biotechnology, Vol.10(57): 12281"12290.

Pohl, S., R. Goddard, and S. Kubik. 2001. A New Cyclic Tetrapeptide Composed of Alternating L-Proline and 3-Aminobenzoic Acid Subunits. Tetrahedron Letters, Vol.42: 7555-7558.

Kitchen, D.B., et al. 2004. Docking and Scoring in Virtual Screening for Drug Discovery: Methods and Applications. Nature Reviews: Drug Discovery, Vol.3(11): 935-49.

Alonso, H., A.A. Bliznyuk, and J.E. Gready. 2006. Combining Docking and Molecular Dynamic Simulations in Drug Design. Medicinal Research Reviews, Vol.26(5):531-568.

Nurbaiti, S. 2009. The Role of Interface Domain Interactions on Thermal Stability of DNA Polymerase I ITB-1. International Journal of Integrative Biology. ISSN 0973-8363. 